\title{
On a classical limit of $q$-deformed Whittaker functions
}

\author{
Anton Gerasimov, Dimitri Lebedev and Sergey Oblezin
}

\begin{abstract}
We provide a derivation of the Givental integral representation of the classical $\mathfrak{g l}_{\ell+1^{-}}$ Whittaker function as a limit $q \rightarrow 1$ of the $q$-deformed $\mathfrak{g l}_{\ell+1}$-Whittaker function represented as a sum over the Gelfand-Zetlin patterns.
\end{abstract}

\section{Introduction}

The $q$-deformed $\mathfrak{g l}_{\ell+1}$-Whittaker functions can be defined as eigenfunctions of the $q$-deformed $\mathfrak{g l}_{\ell+1^{-}}$ Toda chains $[\mathrm{Ru}]$, Et]. Among various eigenfunctions there exists a special class of eigenfunctions with the support in the positive Weyl chamber. By analogy with the classical case we call such functions the class one $q$-deformed $\mathfrak{g l}_{\ell+1}$-Whittaker functions. In GLO1 an explicit representation of the class one $q$-deformed $\mathfrak{g l}_{\ell+1}$-Whittaker function as a sum over the Gelfand-Zetlin patterns was proposed. This representation has remarkable integrality and positivity properties. Precisely each term in the sum is a positive integer multiplied by a weight factor $q^{w t}$ and a character of the torus $U_{1}^{\ell+1}$. This allows to represent the $q$-deformed $\mathfrak{g l}_{\ell+1}$-Whittaker function as a character of a $\mathbb{C}^{*} \times U_{\ell+1^{-}}$-module (i.e. it allows a categorification). The interpretation of the $q$-deformed $\mathfrak{g l}_{\ell+1^{-}}$ Whittaker function as a character shall be considered as a $q$-version of the Shintani-CasselmanShalika formula [Sh, [CS]. Indeed in the limit $q \rightarrow 0$ the $q$-deformed $\mathfrak{g l}_{\ell+1}$-Whittaker function can be identified with the non-Archimedean Whittaker function and the representation of the $q$ deformed Whittaker function as a character reduces to the standard Shintani-Casselman-Shalika formula for non-Archimedean Whittaker function [Sh], CS].

In the limit $q \rightarrow 1$ the $q$-Whittaker functions reproduces the classical Whittaker functions. It was pointed out in GLO1 that in this limit an explicit sum type representation of the class one $q$-deformed $\mathfrak{g l}_{\ell+1}$-Whittaker function turns into the Givental integral representation for the class one $\mathfrak{g l}_{\ell+1}$-Whittaker function Gi] (see also GKLO]). Thus the Givental integral representation shall be considered as the Archimedean counterpart of the Shintani-Casselman-Shalika formula (for more details on this interpretation see GLO2, GLO3, G]). In this note we provide a precise description of the $q \rightarrow 1$ limit reducing the $q$-deformed $\mathfrak{g l}_{\ell+1}$-Whittaker function to its classical analog and explicitly demonstrate that the Givental integral representation arises as a limit of the sum representation of the $q$-deformed $\mathfrak{g l}_{\ell+1}$-Whittaker function. This result is given by Theorem 3.1. The established relation between a sum over the Gelfand-Zetlin patterns for $\mathfrak{g l}_{\ell+1}$ and the Givental integrals for $\mathfrak{g l}_{\ell+1}$ is a special case of a general relation between the Gelfand-Zetlin patterns and the Givental type integrals for classical series of Lie algebras [GLO4]. This relation elucidates the identification of the Givental and the Gelfand-Zetlin graphs noticed in [GLO4]. The relation between the Gelfand-Zetlin and Givental constructions described in this note should be also compared with the duality type relation introduced in GLO5]. We are going to discuss the 
general form of the relation between the Gelfand-Zetlin and the Givental constructions for classical Lie algebras elsewhere.

Acknowledgments: The authors are grateful to A. Borodin and G. Olshanski for their interest in this work. The research was supported by Grant RFBR-09-01-93108-NCNIL-a. AG was also partly supported by Science Foundation Ireland grant. The research of SO was partially supported by P. Deligne's 2004 Balzan Prize in Mathematics.

\section{$1 \quad q$-deformed $\mathfrak{g l}_{\ell+1}$-Whittaker function}

In this Section we recall the explicit construction of the class one $q$-deformed $\mathfrak{g l}_{\ell+1}$-Whittaker functions derived in [GLO1]. Quantum $q$-deformed $\mathfrak{g l}_{\ell+1}$-Toda chain (see e.g. [Ru], [Et]) is defined by a set of $\ell+1$ mutually commuting functionally independent quantum Hamiltonians $\mathcal{H}_{r}^{\mathfrak{g l}}{ }_{\ell+1}$, $r=1, \ldots, \ell+1$ :

$$
\left.\mathcal{H}_{r}^{\mathfrak{g l}} \underline{p}_{\ell+1}\right)=\sum_{I_{r}}\left(\widetilde{X}_{i_{1}}^{1-\delta_{i_{2}-i_{1}, 1}} \cdot \ldots \cdot \widetilde{X}_{i_{r-1}}^{1-\delta_{i_{r}-i_{r-1}, 1}} \cdot \widetilde{X}_{i_{r}}^{1-\delta_{i_{r+1}-i_{r}, 1}}\right) T_{i_{1}} \cdot \ldots \cdot T_{i_{r}}
$$

where $r=1, \ldots, \ell+1$ and $i_{r+1}=\ell+2$. The summation in (1.1) goes over all ordered subsets $I_{r}=\left\{i_{1}<i_{2}<\cdots<i_{r}\right\}$ of $\{1,2, \cdots, \ell+1\}$. Here we use the notations

$$
\begin{gathered}
T_{i} f\left(\underline{p}_{\ell+1}\right)=f\left(\underline{\widetilde{p}}_{\ell+1}\right), \quad \widetilde{p}_{\ell+1, k}=p_{\ell+1, k}+\delta_{k, i}, \\
\widetilde{X}_{i}=1-q^{p_{\ell+1, i}-p_{\ell+1, i+1}+1}, \quad i=1, \ldots, \ell, \quad \widetilde{X}_{\ell+1}=1 .
\end{gathered}
$$

The corresponding eigenvalue problem can be written in the following form:

$$
\left.\left.\mathcal{H}_{r}^{\mathfrak{g l} l_{\ell+1}}\left(\underline{p}_{\ell+1}\right) \Psi_{z_{1}, \ldots, z_{\ell+1}}^{\mathfrak{g l}_{\ell+1}} \underline{p}_{\ell+1}\right)=\left(\sum_{I_{r}} \prod_{i \in I_{r}} z_{i}\right) \Psi_{z_{1}, \ldots, z_{\ell+1}}^{\mathfrak{g l}_{\ell+1}} \underline{p}_{\ell+1}\right),
$$

and the first nontrivial Hamiltonian is given by

$$
\mathcal{H}_{1}^{\mathfrak{g l} l_{\ell+1}}\left(\underline{p}_{\ell+1}\right)=\sum_{i=1}^{\ell}\left(1-q^{p_{\ell+1, i}-p_{\ell+1, i+1}+1}\right) T_{i}+T_{\ell+1} .
$$

One of the main results of [GLO1] now can be formulated as follows. Given $\underline{p}_{\ell+1}=\left(p_{\ell+1,1}, \ldots, p_{\ell+1, \ell+1}\right)$

let us denote by $\mathcal{P}^{(\ell+1)}\left(\underline{p}_{\ell+1}\right)$ a set of collections of the integer parameters $p_{k, i}, k=1, \ldots, \ell$, $i=1, \ldots, k$ satisfying the Gelfand-Zetlin conditions $p_{k+1, i} \geq p_{k, i} \geq p_{k+1, i+1}$. Let $\mathcal{P}_{\ell+1, \ell}\left(\underline{p}_{\ell+1}\right)$ be a set of $\underline{p}_{\ell}=\left(p_{\ell, 1}, \ldots, p_{\ell, \ell}\right), p_{\ell, i} \in \mathbb{Z}$, satisfying the conditions $p_{\ell+1, i} \geq p_{\ell, i} \geq p_{\ell+1, i+1}$.

Theorem 1.1 A common solution of the eigenvalue problem (1.2) can be written in the following form. For $\underline{p}_{\ell+1}$ being in the dominant domain $p_{\ell+1,1} \geq \ldots \geq p_{\ell+1, \ell+1}$, the solution is given by

$$
\begin{gathered}
\left.\Psi_{z_{1}, \ldots, z_{\ell+1}}^{\mathfrak{g l}_{\ell+1}} \underline{p}_{\ell+1}\right)=\sum_{p_{k, i} \in \mathcal{P}^{(\ell+1)}\left(\underline{p}_{\ell+1}\right)} \prod_{k=1}^{\ell+1} z_{k}^{\sum_{i} p_{k, i}-\sum_{i} p_{k-1, i}} \\
\times \frac{\prod_{k=2}^{\ell} \prod_{i=1}^{k-1}\left(p_{k, i}-p_{k, i+1}\right)_{q} !}{\prod_{k=1}^{\ell} \prod_{i=1}^{k}\left(p_{k+1, i}-p_{k, i}\right)_{q} !\left(p_{k, i}-p_{k+1, i+1}\right)_{q} !}
\end{gathered}
$$


where we use the notation $(n)_{q} !=(1-q) \ldots\left(1-q^{n}\right)$. When $\underline{p}_{\ell+1}$ is outside the dominant domain we set

$$
\Psi_{z_{1}, \ldots, z_{\ell+1}}^{\mathfrak{g} l_{\ell+1}}\left(p_{\ell+1,1}, \ldots, p_{\ell+1, \ell+1}\right)=0 .
$$

Example 1.1 Let $\mathfrak{g}=\mathfrak{g l}_{2}, p_{2,1}:=p_{1} \in \mathbb{Z}, p_{2,2}:=p_{2} \in \mathbb{Z}$ and $p_{1,1}:=p \in \mathbb{Z}$. The function

$$
\begin{gathered}
\Psi_{z_{1}, z_{2}}^{\mathfrak{g} l_{2}}\left(p_{1}, p_{2}\right)=\sum_{p_{2} \leq p \leq p_{1}} \frac{z_{1}^{p} z_{2}^{p_{1}+p_{2}-p}}{\left(p_{1}-p\right)_{q} !\left(p-p_{2}\right)_{q} !}, \quad p_{1} \geq p_{2}, \\
\Psi_{z_{1}, z_{2}}^{\mathfrak{g} l_{2}}\left(p_{1}, p_{2}\right)=0, \quad p_{1}<p_{2},
\end{gathered}
$$

is a common eigenfunction of mutually commuting Hamiltonians

$$
\mathcal{H}_{1}^{\mathfrak{g l} l_{2}}=\left(1-q^{p_{1}-p_{2}+1}\right) T_{1}+T_{2}, \quad \mathcal{H}_{2}^{\mathfrak{g} l_{2}}=T_{1} T_{2} .
$$

The formula (1.4) can be easily rewritten in the recursive form.

Corollary 1.1 The following recursive relation holds

$$
\left.\Psi_{z_{1}, \ldots, z_{\ell+1}}^{\mathfrak{g l}_{\ell+1}}\left(\underline{p}_{\ell+1}\right)=\sum_{\left.\underline{p}_{\ell} \in \mathcal{P}_{\ell+1, \ell} \underline{p}_{\ell+1}\right)} \Delta\left(\underline{p}_{\ell}\right) z_{\ell+1}^{\sum_{i} p_{\ell+1, i}-\sum_{i} p_{\ell, i}} Q_{\ell+1, \ell}\left(\underline{p}_{\ell+1}, \underline{p}_{\ell} \mid q\right) \Psi_{z_{1}, \ldots, z_{\ell}}^{\mathfrak{g l}_{\ell}} \underline{p}_{\ell}\right),
$$

where

$$
\begin{gathered}
Q_{\ell+1, \ell}\left(\underline{p}_{\ell+1}, \underline{p}_{\ell} \mid q\right)=\frac{1}{\prod_{i=1}^{\ell}\left(p_{\ell+1, i}-p_{\ell, i}\right)_{q} !\left(p_{\ell, i}-p_{\ell+1, i+1}\right)_{q} !} \\
\Delta\left(\underline{p}_{\ell}\right)=\prod_{i=1}^{\ell-1}\left(p_{\ell, i}-p_{\ell, i+1}\right)_{q} !
\end{gathered}
$$

The following representations of the class one $q$-deformed $\mathfrak{g l}_{\ell+1}$-Whittaker function are a consequence of the positivity and integrality of the coefficients of the $q$-series expansions of each term in the sum (1.4) (see GLO1 for details).

Proposition 1.1 (i). There exists a $\mathbb{C}^{*} \times G L_{\ell+1}(\mathbb{C})$ module $V$ such that the common eigenfunction (1.4) of the $q$-deformed Toda chain allows the following representation for $p_{\ell+1,1} \geq p_{\ell+1,2} \geq \ldots \geq$ $p_{\ell+1, \ell+1}$ :

$$
\Psi_{\underline{\lambda}}^{\mathfrak{g l}_{\ell+1}}\left(\underline{p}_{\ell+1}\right)=\operatorname{Tr}_{V} q^{L_{0}} \prod_{i=1}^{\ell+1} q^{\lambda_{i} H_{i}},
$$

where $H_{i}:=E_{i, i}, i=1, \ldots, \ell+1$ are Cartan generators of $\mathfrak{g l}_{\ell+1}=\operatorname{Lie}\left(G L_{\ell+1}\right)$ and $L_{0}$ is a generator of $\operatorname{Lie}\left(\mathbb{C}^{*}\right)$.

(ii). There exists a finite-dimensional $\mathbb{C}^{*} \times G L_{\ell+1}(\mathbb{C})$ module $V_{f}$ such that the following representation holds for $p_{\ell+1,1} \geq p_{\ell+1,2} \geq \ldots \geq p_{\ell+1, \ell+1}$ :

$$
\widetilde{\Psi}_{\underline{\lambda}}^{\mathfrak{g l} \ell+1}\left(\underline{p}_{\ell+1}\right)=\Delta\left(\underline{p}_{\ell+1}\right) \Psi_{\underline{\lambda}}^{\mathfrak{g l} \mathfrak{l}_{\ell+1}}\left(\underline{p}_{\ell+1}\right)=\operatorname{Tr}_{V_{f}} q^{L_{0}} \prod_{i=1}^{\ell+1} q^{\lambda_{i} H_{i}}
$$

The module $V$ entering (1.7) and the module $V_{f}$ entering (1.8) have a structure of modules under the action of (quantum) affine Lie algebras [GLO1]. 


\section{Classical limit of $q$-deformed Toda chain}

In this Section we define a limit $q \rightarrow 1$ of the $q$-deformed $\mathfrak{g l}_{\ell+1}$-Toda chain reproducing the standard $\mathfrak{g l}_{\ell+1}$-Toda chain. We provide an explicit check that the first two generators of the ring of quantum Hamiltonians of $\mathfrak{g l}_{\ell+1}$-Toda chain arise as a limit of some combinations of the following quantum Hamiltonians of the $q$-deformed Toda chain

$$
\begin{gathered}
\mathcal{H}_{1}^{\mathfrak{g} \mathfrak{l}_{\ell+1}}\left(\underline{p}_{\ell+1} \mid q\right)=\sum_{i=1}^{\ell}\left(1-q^{p_{\ell+1, i}-p_{\ell+1, i+1}+1}\right) T_{i}+T_{\ell+1}, \\
\mathcal{H}_{\ell+1}^{\mathfrak{g l}_{\ell+1}}\left(\underline{p}_{\ell+1} \mid q\right)=T_{1} T_{2} \cdots T_{\ell+1} .
\end{gathered}
$$

Let us introduce the following parametrization:

$$
q=e^{-\epsilon}, \quad p_{\ell+1, k}=(\ell+2-2 k) m(\epsilon)+x_{\ell+1, k} \epsilon^{-1} .
$$

Here $m(\epsilon) \in \mathbb{Z}$ is given by

$$
m(\epsilon)=-\left[\epsilon^{-1} \ln \epsilon\right]
$$

and $[x] \in \mathbb{Z}$ is the integer part of $x$.

Proposition 2.1 The following limiting relations hold:

$$
\begin{gathered}
\left.\left.H_{1}^{\mathfrak{g l}} \underline{\operatorname{l}}_{\ell+1} \underline{x}_{\ell+1}\right)=\lim _{\epsilon \rightarrow 0} \frac{1}{\epsilon}\left[\mathcal{H}_{1}^{\mathfrak{g l}} \underline{p}_{\ell+1}(x, \epsilon) \mid q(\epsilon)\right)-(\ell+1)\right], \\
H_{2}^{\mathfrak{g l}_{\ell+1}}\left(\underline{x}_{\ell+1}\right)=-\lim _{\epsilon \rightarrow 0} \frac{1}{\epsilon^{2}}\left[\mathcal{H}_{1}^{\mathfrak{g} l_{\ell+1}}\left(\underline{p}_{\ell+1}(x, \epsilon) \mid q(\epsilon)\right)-\mathcal{H}_{\ell+1}^{\mathfrak{g l}}\left(\underline{p}_{\ell+1}(x, \epsilon) \mid q(\epsilon)\right)-\ell\right. \\
\left.\left.+\frac{1}{2}\left(\mathcal{H}_{\ell+1}^{\mathfrak{g l}} \underline{\underline{p}}_{\ell+1}(x, \epsilon) \mid q(\epsilon)\right)-1\right)^{2}\right]
\end{gathered}
$$

where $H_{i}^{\mathfrak{g l}_{\ell+1}}, i=1,2$ are the standard quantum Hamiltonians of the $\mathfrak{g l}_{\ell+1}$-Toda chain:

$$
\begin{gathered}
H_{1}^{\mathfrak{g l}_{\ell+1}}\left(\underline{x}_{\ell+1}\right)=\sum_{i=1}^{\ell+1} \frac{\partial}{\partial x_{\ell+1, i}}, \\
H_{2}^{\mathfrak{g l} l_{\ell+1}}\left(\underline{x}_{\ell+1}\right)=-\frac{1}{2} \sum_{i=1}^{\ell+1} \frac{\partial^{2}}{\partial x_{i}^{2}}+\sum_{i=1}^{\ell} e^{x_{i+1}-x_{i}} .
\end{gathered}
$$

Proof. Using the fact that $\exp \left(\epsilon\left[2(\epsilon)^{-1} \ln (\epsilon)\right]=\epsilon^{2}\left(1+O\left(\epsilon^{2} / \ln \epsilon\right)\right)\right.$ we have

$$
\begin{gathered}
\left.\mathcal{H}_{1}^{\mathfrak{g l}} \underline{p}_{\ell+1} \mid q\right)=(\ell+1)+\epsilon \sum_{i=1}^{\ell+1} \frac{\partial}{\partial x_{\ell+1, i}}+\epsilon^{2}\left(\frac{1}{2} \sum_{i=1}^{\ell+1} \frac{\partial^{2}}{\partial x_{\ell+1, i}^{2}}-\sum_{k=1}^{\ell} e^{x_{\ell+1, k+1}-x_{\ell+1, k}}\right)+O\left(\epsilon^{3}\right), \\
\mathcal{H}_{\ell+1}^{\mathfrak{g l}_{\ell+1}}\left(\underline{p}_{\ell+1} \mid q\right)=1+\epsilon \sum_{i=1}^{\ell+1} \frac{\partial}{\partial x_{\ell+1, i}}+\frac{1}{2} \epsilon^{2} \sum_{i, j=1}^{\ell+1} \frac{\partial^{2}}{\partial x_{\ell+1, i} \partial x_{\ell+1, j}}+O\left(\epsilon^{3}\right) .
\end{gathered}
$$


Now the limiting formulas can be straightforwardly verified. We have

$$
\begin{aligned}
\mathcal{H}_{1}^{\mathfrak{g l}_{\ell+1}}\left(\underline{p}_{\ell+1} \mid q\right)-\mathcal{H}_{\ell+1}^{\mathfrak{g l}_{\ell+1}}\left(\underline{p}_{\ell+1} \mid q\right)-\ell=\epsilon^{2}\left(-\frac{1}{2} \sum_{i \neq j}^{\ell+1} \frac{\partial^{2}}{\partial x_{\ell+1, i} \partial x_{\ell+1, j}}-\sum_{k=1}^{\ell} e^{x_{\ell+1, k+1}-x_{\ell+1, k}}\right)+O\left(\epsilon^{3}\right), \\
\frac{1}{2}\left(\mathcal{H}_{\ell+1}^{\mathfrak{g l} \underline{l}_{\ell+1}}\left(\underline{p}_{\ell+1} \mid q\right)-1\right)^{2}=\frac{1}{2} \epsilon^{2}\left(\sum_{i, j=1}^{\ell+1} \frac{\partial^{2}}{\partial x_{\ell+1, i} \partial x_{\ell+1, j}}\right)+O\left(\epsilon^{3}\right),
\end{aligned}
$$

and thus

$$
\begin{gathered}
\mathcal{H}_{1}^{\mathfrak{g l} \ell+1}\left(\underline{p}_{\ell+1} \mid q\right)-\mathcal{H}_{\ell+1}^{\mathfrak{g l}}\left(\underline{p}_{\ell+1} \mid q\right)-\ell+\frac{1}{2}\left(\mathcal{H}_{\ell+1}^{\mathfrak{g l}}\left(\underline{p}_{\ell+1} \mid q\right)-1\right)^{2} \\
=\epsilon^{2}\left(\frac{1}{2} \sum_{i=1}^{\ell+1} \frac{\partial^{2}}{\partial x_{\ell+1, i}^{2}}-\sum_{k=1}^{\ell} e^{x_{\ell+1, k+1}-x_{\ell+1, k}}\right)+O\left(\epsilon^{3}\right) .
\end{gathered}
$$

It is easy to see that the eigenfunction problem (1.2) is transformed into the standard eigenfunction problem if we use the following parametrization of the spectral variables $z_{i}=e^{\imath \epsilon \lambda_{i}}, i=$ $1, \ldots, \ell+1$.

\section{Classical limit of class one Whittaker function}

In the limit $q \rightarrow 1$ defined in the previous Section the class one solution (1.4) of the $q$-deformed $\mathfrak{g l}_{\ell+1}$-Toda chain should goes to the class one solution of the classical $\mathfrak{g l}_{\ell+1}$-Toda chain. In the classical setting an integral representation for class one $\mathfrak{g l}_{\ell+1}$-Whittaker function was constructed by Givental [Gi], (see [GKLO] for a choice of the contour realizing class one condition)

$$
\psi_{\lambda_{1}, \ldots, \lambda_{\ell+1}}^{\mathfrak{g} \mathfrak{l}_{\ell+1}}\left(x_{1}, \ldots, x_{\ell+1}\right)=\int_{C} \prod_{k=1}^{\ell} d \underline{x}_{k} e^{\mathcal{F}^{\mathfrak{g} \mathfrak{l}_{\ell+1}}(x)},
$$

and the function $\mathcal{F}^{\mathfrak{g l}_{\ell+1}}(x)$ is given by

$$
\mathcal{F}^{\mathfrak{g l}_{\ell+1}}(x)=\imath \sum_{n=1}^{\ell+1} \lambda_{n}\left(\sum_{i=1}^{n} x_{n, i}-\sum_{i=1}^{n-1} x_{n-1, i}\right)-\sum_{k=1}^{\ell} \sum_{i=1}^{k}\left(e^{x_{k, i}-x_{k+1, i}}+e^{x_{k+1, i+1}-x_{k, i}}\right) .
$$

Here $C \subset N_{+}$is a small deformation of the subspace $\mathbb{R}^{\frac{(\ell+1) \ell}{2}} \subset \mathbb{C}^{\frac{(\ell+1) \ell}{2}}$ making the integral (3.2) convergent. Besides, we use the following notation: $\underline{\lambda}=\left(\lambda_{1}, \ldots, \lambda_{\ell+1}\right) ; x_{i}:=x_{\ell+1, i}, i=1, \ldots, \ell+1$.

The integral representation (3.1) allows a recursive presentation analogous to (1.5)

$$
\psi_{\lambda_{1}, \ldots, \lambda_{\ell+1}}^{\mathfrak{g} \mathfrak{l}_{\ell+1}}\left(x_{1}, \ldots, x_{\ell+1}\right)=\int_{\mathbb{R}^{\ell}} d \underline{x}_{\ell} Q_{\mathfrak{g l}_{\ell}}^{\mathfrak{g l} \mathfrak{l}_{\ell+1}}\left(\underline{x}_{\ell+1} ; \underline{x}_{\ell} ; \lambda_{\ell+1}\right) \psi_{\lambda_{1}, \ldots, \lambda_{\ell}}^{\mathfrak{g l}_{\ell}}\left(x_{\ell, 1}, \ldots, x_{\ell \ell}\right),
$$

where

$$
\begin{gathered}
Q_{\mathfrak{g l}_{\ell}}^{\mathfrak{g l}_{\ell+1}}\left(\underline{x}_{\ell+1} ; \underline{x}_{\ell} ; \lambda_{\ell+1}\right)=\exp \left\{\imath \lambda_{\ell+1}\left(\sum_{i=1}^{\ell+1} x_{\ell+1, i}-\sum_{i=1}^{\ell} x_{\ell, i}\right)\right. \\
\left.-\sum_{i=1}^{\ell}\left(e^{x_{\ell, i}-x_{\ell+1, i}}+e^{x_{\ell+1, i+1}-x_{\ell, i}}\right)\right\}
\end{gathered}
$$


and we assume $Q_{\mathfrak{g l}_{0}}^{\mathfrak{g l}_{1}}\left(x_{11} ; \lambda_{1}\right)=e^{\imath \lambda_{1} x_{11}}$.

In the following we demonstrate that in the previously defined limit $q \rightarrow 1$ the class one $q$ deformed $\mathfrak{g l}_{\ell+1}$-Whittaker function given by the sum (1.4) indeed turns into the classical class one $\mathfrak{g l}_{\ell+1}$-Whittaker function given by the integral representation (3.1). In particular iterative formula (1.5) turns into (3.3) . For this purpose we need the following asymptotic of the $q$-factorials entering (1.4).

Lemma 3.1 Let us introduce the following functions

$$
f_{\alpha}(y, \epsilon)=(y / \epsilon+\alpha m(\epsilon))_{q} !, \quad \alpha=1,2,
$$

where $m(\epsilon)=-\left[\epsilon^{-1} \ln \epsilon\right], q=e^{-\epsilon}$. Then for $\epsilon \rightarrow+0$ the following expansions hold:

$$
\begin{gathered}
f_{1}(y, \epsilon)=e^{A(\epsilon)+e^{-y}+O(\epsilon)} \\
f_{2}(y, \epsilon)=e^{A(\epsilon)+O\left(\epsilon^{\alpha-1}\right)},
\end{gathered}
$$

where $A(\epsilon)=-\frac{\pi^{2}}{6} \frac{1}{\epsilon}-\frac{1}{2} \ln \frac{\epsilon}{2 \pi}$.

Proof. Taking into account the identity

$$
\ln \prod_{n=1}^{N}\left(1-q^{n}\right)=\sum_{n=1}^{N} \ln \left(1-q^{n}\right)=-\sum_{n=1}^{N} \sum_{r=1}^{+\infty} \frac{1}{r} q^{n r}=-\sum_{r=1}^{+\infty} \frac{q^{r}}{r}\left(\frac{1-q^{N r}}{1-q^{r}}\right)
$$

and using the substitution $q=e^{-\epsilon}, N=\epsilon^{-1} y+\alpha m(\epsilon)$ we obtain

$$
\ln f_{\alpha}(y, \epsilon)=-\sum_{r=1}^{+\infty} \frac{e^{-r \epsilon}}{r}\left(\frac{1-e^{-\alpha r \epsilon m(\epsilon)} e^{-r y}}{1-e^{-r \epsilon}}\right) .
$$

Now expanding the denominator over small $\epsilon$ we have

$$
\ln f_{\alpha}(y, \epsilon)=-\sum_{r=1}^{+\infty} \frac{e^{-r \epsilon}}{r}\left(\frac{1-\epsilon^{\alpha r} e^{-r y}}{1-e^{-r \epsilon}}\right)+\cdots=-\sum_{r=1}^{+\infty} \frac{e^{-r \epsilon}}{r^{2} \epsilon}\left(\frac{1-\epsilon^{\alpha r} e^{-r y}}{1-\frac{1}{2} r \epsilon+\frac{1}{3 !} r^{2} \epsilon^{2}+\cdots}\right)+\cdots
$$

and for the derivative we obtain

$$
\partial_{y} \ln f_{\alpha}(y, \epsilon)=-\sum_{r=1}^{+\infty} \frac{1}{r \epsilon}\left(\frac{\epsilon^{\alpha r} e^{-r y-r \epsilon}}{1-\frac{1}{2} r \epsilon+\frac{1}{3 !} r^{2} \epsilon^{2}+\cdots}\right)+\cdots=\sum_{k=-1}^{+\infty} c_{k} I_{\alpha, k}(y, \epsilon),
$$

where

$$
I_{\alpha, k}(y, \epsilon)=\sum_{r=1}^{+\infty} \epsilon^{k+\alpha r} r^{k} e^{-y r}=\epsilon^{k} \sum_{r=1}^{+\infty} t^{r} r^{k}, \quad t=e^{-y} \epsilon^{\alpha},
$$

and $c_{-1}=-1$. Let us separately analyze the term $I_{\alpha,-1}$ and the other terms $I_{\alpha, k \geq 0}$. We have

$$
I_{\alpha, k \geq 0}(y, \epsilon)=\epsilon^{k}\left(t \frac{\partial}{\partial t}\right)^{k} \frac{1}{1-t}=\epsilon^{k} \frac{\partial^{k}}{\partial y^{k}} \frac{1}{1-\epsilon^{\alpha} e^{-y}},
$$

and thus

$$
I_{\alpha, k \geq 0}=\epsilon^{k+\alpha} e^{-y}+\cdots, \quad \alpha=1,2 .
$$


Now consider the case of $k=-1$

$$
\left.c_{-1} I_{\alpha,-1}(y, \epsilon)=-\frac{1}{\epsilon} \sum_{r=1}^{+\infty} \frac{t^{r}}{r}=-\frac{1}{\epsilon} \ln (1-t)=-\frac{1}{\epsilon} \ln \left(1-\epsilon^{\alpha} e^{-y}\right)\right)=\epsilon^{\alpha-1} e^{-y}+\cdots, \quad t=e^{-y} \epsilon^{\alpha} .
$$

This gives (3.5), (3.6) with an unknown $A(\epsilon)$. To calculate $A(\epsilon)$ we take $e^{-y}=0$ and notice that the resulting function does not depend on $\alpha$. Thus we should calculate the asymptotic of the following function:

$$
\begin{aligned}
\left.\ln f_{\alpha}(y, \epsilon)\right|_{e^{y}=0}= & -\left.\sum_{r=1}^{+\infty} \frac{e^{-r \epsilon}}{r}\left(\frac{1-\epsilon^{r \alpha} e^{-r y}}{1-e^{-r \epsilon}}\right)\right|_{e^{y}=0}=-\sum_{r=1}^{+\infty} \frac{1}{r}\left(\frac{e^{-r \epsilon}}{1-e^{-r \epsilon}}\right)= \\
& =-\sum_{n=1}^{+\infty} \sum_{r=1}^{+\infty} \frac{1}{r} e^{-n r \epsilon}=\ln \prod_{n=1}^{+\infty}\left(1-e^{-n \epsilon}\right) .
\end{aligned}
$$

It can be easily done using the modular properties

$$
\eta\left(-\tau^{-1}\right)=\sqrt{-\imath \tau} \eta(\tau)
$$

of the Dedekind eta function

$$
\eta(\tau)=e^{\frac{\imath \pi \tau}{12}} \prod_{n=1}^{\infty}\left(1-e^{2 \pi \imath n \tau}\right)
$$

Namely, taking $\tau=\frac{\imath \epsilon}{2 \pi}$ we have

$$
\left.f_{\alpha}(y ; \epsilon)\right|_{e^{-y}=0}=\sqrt{2 \pi \epsilon^{-1}} e^{-\frac{\pi^{2}}{6} \epsilon^{-1}} \prod_{n=1}^{\infty}\left(1-e^{-\epsilon^{-1}(2 \pi)^{2} n}\right) .
$$

This allows to infer the following result for the leading coefficients in the asymptotic expansion of $\left.\ln f_{\alpha}(y, \epsilon)\right|_{e^{-y}=0}$ :

$$
A(\epsilon)=-\frac{1}{2} \ln \frac{\epsilon}{2 \pi}-\frac{\pi^{2}}{6} \epsilon^{-1}, \quad \epsilon \longrightarrow+0 .
$$

This completes the proof of Lemma.

Theorem 3.1 Let us use the following parametrization

$$
q=e^{-\epsilon}, \quad p_{\ell+1, k}=(\ell+2-2 k) m(\epsilon)+\epsilon^{-1} x_{\ell+1, k}, \quad z_{k}=e^{\imath \epsilon \lambda_{k}},
$$

where $k=1, \ldots, \ell+1, m(\epsilon)=-\left[\epsilon^{-1} \ln \epsilon\right]$. The integral representation (3.1) of the classical $\mathfrak{g l}_{\ell+1}$-Whittaker function is given by the following limit of the q-deformed class one $\mathfrak{g l}_{\ell+1}$-Whittaker function represented as a sum (1.4)

$$
\left.\psi_{\lambda_{1}, \ldots, \lambda_{\ell+1}}^{\mathfrak{g l}_{\ell+1}}\left(x_{1}, \ldots, x_{\ell+1}\right)=\lim _{\epsilon \rightarrow+0}\left[\epsilon^{\frac{\ell(\ell+1)}{2}} e^{\frac{\ell(\ell+3)}{2} A(\epsilon)} \Psi_{z_{1}, \ldots, z_{\ell+1}}^{\mathfrak{g l}_{\ell+1}} \underline{p}_{\ell+1}\right)\right],
$$

where $A(\epsilon)=-\frac{\pi^{2}}{6} \frac{1}{\epsilon}-\frac{1}{2} \ln \frac{\epsilon}{2 \pi}$ and $x_{i}=x_{\ell+1, i}, i=1, \ldots, \ell+1$. 
Proof. We prove (3.9) by relating the recursive relation (1.5)

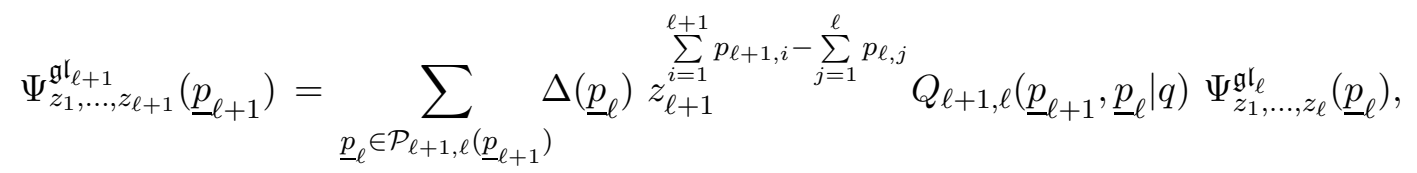

where

$$
\begin{gathered}
Q_{\ell+1, \ell}\left(\underline{p}_{\ell+1}, \underline{p}_{\ell} \mid q\right)=\frac{1}{\prod_{i=1}^{\ell}\left(p_{\ell+1, i}-p_{\ell, i}\right)_{q} !\left(p_{\ell, i}-p_{\ell+1, i+1}\right)_{q} !}, \\
\Delta\left(\underline{p}_{\ell}\right)=\prod_{i=1}^{\ell-1}\left(p_{\ell, i}-p_{\ell, i+1}\right)_{q} !,
\end{gathered}
$$

with the recursive relation (3.3) for the classical Whittaker function.

Let us introduce the following parametrization of the elements of the Gelfand-Zetlin patterns $\underline{p}_{\ell} \in \mathcal{P}_{\ell+1, \ell}\left(\underline{p}_{\ell+1}\right)$ :

$$
p_{\ell, k}=\epsilon^{-1} x_{\ell, k}+a_{k} m(\epsilon), \quad m(\epsilon)=-\left[\epsilon^{-1} \ln \epsilon\right],
$$

where $a_{k}$ are some constants. The Gelfand-Zetlin conditions on weights $\underline{p}_{\ell+1}$ reads as follows:

$$
p_{\ell+1, k} \geq p_{\ell, k} \geq p_{\ell+1, k+1}, \quad k=1, \ldots, \ell,
$$

and they lead to

$$
\epsilon^{-1} x_{\ell+1, k}+(\ell+2-2 k) m(\epsilon) \geq \epsilon^{-1} x_{\ell, k}+a_{k} m(\epsilon) \geq \epsilon^{-1} x_{\ell+1, k+1}+(\ell-2 k) m(\epsilon) .
$$

The requirement that the limit $\epsilon \rightarrow+0$ preserves the conditions (3.12) implies the following restrictions on the parameters $a_{k}$ :

$$
\ell-2 k+2>a_{k}>\ell-2 k, \quad k=1, \ldots, \ell .
$$

Since $\underline{p}_{\ell}=\left(p_{\ell, 1}, \ldots, p_{\ell, \ell}\right) \in \mathbb{Z}^{\ell}$ the only consistent choice in the limit $\epsilon \rightarrow+0$ is $a_{k}=\ell+1-2 k$, $k=1, \ldots, \ell$. Although the variables $p_{\ell, k}$ are restricted to be in positive Weyl chamber i.e. $p_{\ell, k} \geq$ $p_{\ell, k+1}$, in the limit $\epsilon \rightarrow+0$ the variables $x_{\ell, k}$ have no such restrictions. This follows from a simple observation that the limit $\epsilon \rightarrow+0$ the $\frac{a}{\epsilon}-b\left[\epsilon^{-1} \ln \epsilon\right] \rightarrow+\infty /-\infty$ depends only on the sign of non-zero coefficient $b$. Thus we have

$$
p_{\ell, k}=\epsilon^{-1} x_{\ell, k}+(\ell+1-2 k) m(\epsilon) .
$$

Now using Lemma 3.1 it is easy to obtain the following limiting formulas:

$$
\begin{gathered}
\lim _{\epsilon \rightarrow+0} e^{2 \ell A(\epsilon)} \mathcal{Q}_{\ell+1, \ell}\left(\underline{p}_{\ell+1}, \underline{p}_{\ell} \mid q\right)=\lim _{\epsilon \rightarrow+0} \frac{e^{2 \ell A(\epsilon)}}{\prod_{i=1}^{\ell} f_{1}\left(x_{\ell+1, i}-x_{\ell, i}, \epsilon\right) f_{1}\left(x_{\ell, i}-x_{\ell+1, i+1}, \epsilon\right)} \\
=\left.Q_{\mathfrak{g l}_{\ell}}^{\mathfrak{g l}_{\ell+1}}\left(\underline{x}_{\ell+1} ; \underline{x}_{\ell} ; \lambda_{\ell+1}\right)\right|_{\lambda_{\ell+1}=0}, \\
\lim _{\epsilon \rightarrow+0} e^{(1-\ell) A(\epsilon)} \Delta\left(\underline{p}_{\ell}\right)=\lim _{\epsilon \rightarrow+0} e^{(1-\ell) A(\epsilon)} \prod_{i=1}^{\ell-1} f_{2}\left(x_{\ell, i}-x_{\ell, i+1}, \epsilon\right)=1,
\end{gathered}
$$


where $Q_{\mathfrak{g l}_{\ell}}^{\mathfrak{g l}_{\ell+1}}\left(\underline{x}_{\ell+1} ; \underline{x}_{\ell} ; \lambda_{\ell+1}\right)$ is given by (3.4). This implies the following identity:

$$
\begin{gathered}
\lim _{\epsilon \rightarrow+0}\left\{\epsilon_{\underline{p}_{\ell} \in \mathcal{P}_{\ell+1, \ell}\left(\underline{p}_{\ell+1}\right)} z_{\ell=1}^{\sum_{\ell=1}^{\ell+1} p_{\ell+1, i}-\sum_{j=1}^{\ell} p_{\ell, j}}\left[e^{(\ell+1) A(\epsilon)} Q_{\ell+1, \ell}\left(\underline{p}_{\ell+1} ; \underline{p}_{\ell} \mid q\right) \Delta\left(\underline{p}_{\ell}\right)\right]\right. \\
\left.\left.\times \epsilon^{\frac{\ell(\ell-1)}{2}} e^{\frac{(\ell-1)(\ell+2)}{2} A(\epsilon)} \Psi_{z_{1}, \ldots, z_{\ell}}^{\mathfrak{g l}_{\ell}} \underline{p}_{\ell}\right)\right\} \\
\times \lim _{\epsilon \rightarrow+0}\left[e^{(\ell+1) A(\epsilon)} \underline{x}_{\ell} \exp \left\{\imath \lambda_{\ell+1}\left(\sum_{i=1}^{\ell+1} x_{\ell+1, i}-\sum_{j=1}^{\ell} x_{\ell, j}\right)\right\}\right. \\
\left.\times \lim _{\epsilon \rightarrow+0}\left[\epsilon^{\frac{\ell(\ell-1)}{2}} e^{\frac{(\ell-1)(\ell+2)}{2} A(\epsilon)} \Psi_{z_{1}, \ldots, z_{\ell}}^{\mathfrak{g l}_{\ell}} \underline{p}_{\ell}\left(\underline{x}_{\ell}, \epsilon\right)\right)\right] .
\end{gathered}
$$

Thus we recover the recursive relations (3.3) for the Givental integrals directly leading to the integral representation (3.1) for the classical $\mathfrak{g l}_{\ell+1}$-Whittaker function. Using (3.17) iteratively over $\ell$ we obtain (3.9).

Example 3.1 For $\ell=1$ we have

$$
\begin{gathered}
\Psi_{z_{1}, z_{2}}^{\mathfrak{g l}_{2}}\left(p_{2,1}, p_{2,2}\right)=\sum_{p_{2,2} \leq p_{1,1} \leq p_{2,1}} \frac{z_{1}^{p_{1,1}} z_{2}^{p_{2,1}+p_{2,2}-p_{1,1}}}{\left(p_{1,1}-p_{2,2}\right)_{q} !\left(p_{2,1}-p_{1,1}\right)_{q} !}, \quad p_{2,2} \leq p_{2,1}, \\
\Psi_{z_{1}, z_{2}}\left(p_{2,1}, p_{2,2}\right)=0, \quad p_{2,2}>p_{2,1} .
\end{gathered}
$$

Using the parametrization

$$
q=e^{-\epsilon}, \quad p_{21}=m(\epsilon)+x_{21} \epsilon^{-1} \quad p_{22}=-m(\epsilon)+x_{21} \epsilon^{-1} \quad z_{i}=e^{\imath \epsilon \lambda_{i}}, \quad i=1,2,
$$

with $m(\epsilon)=-\left[\epsilon^{-1} \ln \epsilon\right]$ we obtain

$$
\Psi_{z_{1}, z_{2}}^{\mathfrak{g l}_{2}}\left(p_{21}, p_{22}\right)=\sum_{x_{22}-\epsilon m(\epsilon) \leq x_{11} \leq x_{2,1}+\epsilon m(\epsilon)} \frac{e^{\imath \lambda_{1} x_{11}+\imath \lambda_{2}\left(x_{21}+x_{22}-x_{11}\right)}}{\left(\left(x_{11}-x_{22}\right) / \epsilon+m(\epsilon)\right)_{q} !\left(\left(x_{21}-x_{11}\right) / \epsilon+m(\epsilon)\right)_{q} !},
$$

where we use the notations $p_{11}=x_{11} / \epsilon$. Taking into account

$$
\frac{1}{(y / \epsilon+m(\epsilon))_{q} !}=e^{+\frac{\pi^{2}}{6} \frac{1}{\epsilon}+\frac{1}{2} \ln \frac{\epsilon}{2 \pi}-e^{-y}+O(\epsilon)}
$$

we obtain

$$
\begin{aligned}
& \psi_{\lambda_{1}, \lambda_{2}}^{\mathfrak{g l}_{2}}\left(x_{1}, x_{2}\right)=\lim _{\epsilon \rightarrow+0} \epsilon e^{-\frac{\pi^{2}}{3} \frac{1}{\epsilon}-\ln \frac{\epsilon}{2 \pi}} \Psi_{z_{1}, z_{2}}^{\mathfrak{g l}_{2}}\left(p_{21}, p_{22}\right) \\
& =\int_{\mathbb{R}} d x_{11} e^{\imath \lambda_{1} x_{11}} e^{\imath \lambda_{2}\left(x_{21}+x_{22}-x_{11}\right)} e^{-e^{x_{11}-x_{21}-e^{x_{22}-x_{11}}}} .
\end{aligned}
$$




\section{References}

[Et] P. Etingof, Whittaker functions on quantum groups and q-deformed Toda operators, Amer. Math. Soc. Transl. Ser.2, 194, 9-25, Amer. Math. Soc., Providence, RI, 1999; [arXiv:math.QA/9901053] .

[CS] W. Casselman, J. Shalika, The unramified principal series of p-adic groups II. The Whittaker function. Comp. Math. 41 (1980) 207-231 .

[GKLO] A. Gerasimov, S. Kharchev, D. Lebedev, S. Oblezin, On a Gauss-Givental representation of quantum Toda chain wave function, Int. Math. Res. Notices, (2006), Aricle ID96489, 23 pages, [arXiv:math.RT/0505310].

[GLO1] A. Gerasimov, D. Lebedev, S. Oblezin, On q-deformed $\mathfrak{g l}_{\ell+1}$-Whittaker functions I,II,III, Comm. Math. Phys. 294 (2010), 97-119, [math.RT/0803.0145]; Comm. Math. Phys. 294 (2010), 121-143, [math.RT/0803.0970]; [math.RT/0805.3754].

[GLO2] A. Gerasimov, D. Lebedev, S. Oblezin, Parabolic Whittaker Functions and Topological Field Theories I, [arXiv:1002.2622].

[GLO3] A. Gerasimov, D. Lebedev and S. Oblezin, From Archimedean L-factors to Topological Field Theories Let. Math. Phys. DOI 10.1007/sl 105-010-0407-3; Mathematische Arbeitstagung, 2009, MPIM 2009-401.

[GLO4] A. Gerasimov, D. Lebedev, S. Oblezin, New integral representations of Whittaker functions for classical groups, [arXiv:math.RT/0705.2886].

[GLO5] A. Gerasimov, D. Lebedev, S. Oblezin, Baxter operator and archimedean Hecke algebras, Commun. Math. Phys. 284(3), (2008), 867-896; [arXiv:0706.3476].

[G] A. Gerasimov, A Quantum Field Theory Model of Archimedean Geometry, talk at Rencontres Itzykson 2010: New trends in quantum integrability, 21-23 June, 2010, IPhT Saclay, France (see link on the webpage of the conference).

[Gi] A. Givental, Stationary Phase Integrals, Quantum Toda Lattices, Flag Manifolds and the Mirror Conjecture. Topics in Singularity Theory, Amer. Math. Soc. Transl. Ser., 2 180, AMS, Providence, Rhode Island, 1997, 103-115 [arXiv:alg-geom/9612001].

[Ru] S. N. M. Ruijsenaars, Relativistic Toda system, Comm. Math. Phys. 133 (1990), 217-247.

[Sh] T. Shintani, On an explicit formula for class 1 Whittaker functions on $G L_{n}$ over p-adic fields. Proc. Japan Acad. 52 (1976), 180-182

A.G. Institute for Theoretical and Experimental Physics, 117259, Moscow, Russia; School of Mathematics, Trinity College Dublin, Dublin 2, Ireland;

Hamilton Mathematics Institute, Trinity College Dublin, Dublin 2, Ireland;

D.L. Institute for Theoretical and Experimental Physics, 117259, Moscow, Russia; E-mail address: lebedev@itep.ru 
S.O. Institute for Theoretical and Experimental Physics, 117259, Moscow, Russia;

E-mail address: Sergey.Oblezin@itep.ru 